\title{
EDITORIAL
}

\section{Molding the Light Flow at Desire}

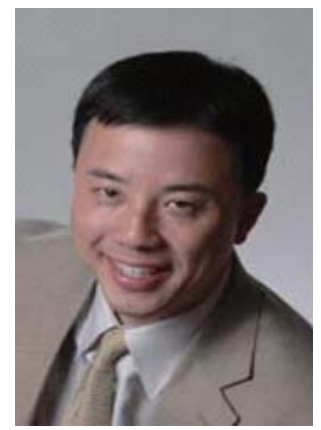

Prof. Xiang ZHANG

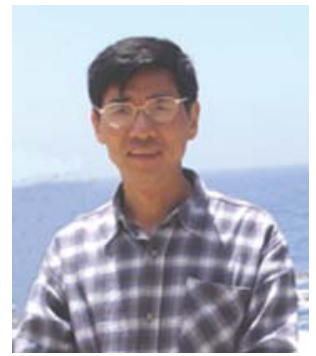

Prof. Shi-ning ZHU

How to manipulate light at will, either in space or in time, is one of the central problems in the optics community. In the past decade, researchers have demonstrated the ability to control light in unprecedented ways with the aid of artificially engineered materials, among which metamaterials have attracted world-wide interest. Metamaterials are composites of subwavelength artificial structures. From effective medium theory, a metamaterial can be characterized as a homogeneous material, whose EM properties (i.e., electric permittivity and magnetic permeability) mainly rely on the internal structures rather than the chemical elements of the metamaterial. Some unusual material properties unavailable in nature, such as optical magnetism and negative refractive index, have been realized by metamaterials. With the full freedom offered by metamaterials, researchers have demonstrated negative refraction, reversed Cherenkov radiation, super lenses, and recent invisibility cloaks, which bring revolutionary impacts on modern optics.

Metamaterials have become a very active research area involving many other disciplines. For instance, at the early stage of metamaterial research, negative refraction and superlensing were concurrently explored in photonic crystals. Nowadays, as metamaterials enter the optical regime, the plasmonic effect of metals can play an important role. Indeed, the investigation on coupling between metallic metamaterial elements and biosensing by metamaterials has been rapidly developing. It is expected that the interplay between the communities of photonic crystals, metamaterials, and plasmonics will continue to stimulate exciting ideas and discoveries.

Frontiers of Physics in China gathers together experts in these three communities for this special issue. Ten excellent papers present recent progress and a future outlook in artificial wave-functional materials. On the subject of photonic crystals, Y. Liu et al. present ultrafast optical switching from Kerr nonlinearity; J. F. Liu and X. H. Wang demonstrate the manipulation of spontaneous emission by photonic crystal or metallic micro-/nanostructures; while recent research on surface mode optical microcavities in photonic crystals is reviewed by J. Wang and M. Qiu. T. R. Zhai et al. introduce the holographic method to fabricate photonic crystals with low refractive index materials and apply it to the design of microlasers. In terms of metamaterials, H. Liu et al. present a review on the hybridization effect due to the coupling between individual metamaterial elements, which is beyond the traditional effective medium theory. J. M. Hao et al. show how to manipulate light polarizations by microwave and optical metamaterials. Two papers focus on transformation optics. One is by Y. Lai et al., who present the remote illusion device that can transform the stereoscopic image of an object into the illusion of another different object; while in the other paper, D. Bao et al. propose a broadband nonmagnetic dielectric cloak. Sub-diffraction-limited far-field imaging in infrared is investigated by P. Chaturvedi and N. X. Fang. Finally, in plasmonics, A. Garcia-Etxarri et al. reports chemical sensing based on plasmonic nanoparticle aggregation.

This special issue summarizes some of the exciting results obtained in the past few years. Without any doubt, we envision that this field will grow, and many more will emerge in the near future.

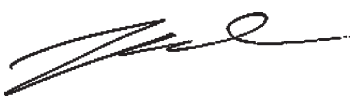

Xiang ZHANG (University of California at Berkeley, USA)

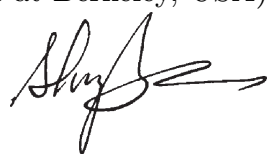

Shi-ning ZHU

(Nanjing University, China)

Guest Editors 